\title{
Análise de temas recorrentes nas dissertações da linha de Informação e Comunicação do programa de Pós-Graduação em Design da UFMA
}

Bruno Serviliano é Doutor em Design pela UNESP-BAURU (2019). Mestre em Design pela UFMA (2014), especialista em Design Gráfico (2009) e graduado em Publicidade e Propaganda e Desenho Industrial. Professor no Departamento de Desenho e Tecnologia da Universidade Federal do Maranhão. Aulas no curso de Design e no programa de Pós em Design da UFMA. Tem como foco de pesquisa Design Gráfico Inclusivo e Artefatos Interativos. <bruno.serviliano@ufma.br> ORCID 0000-0002-5960-5835
Resumo O Design de Informação (D.I.) tem papel fundamental na transmissão de mensagens de qualquer natureza e se torna inerente à cidadania, pois seus elementos e métodos têm consequências diretas na sociedade. A produção científica dessa área ajuda a consolidá-la como campo científico apto a cooperar para o desenvolvimento da sociedade. Considerando a importância do D.I. e de seus impactos, o presente artigo teve como objetivo principal o de descrever e analisar as dissertações da linha de pesquisa de Informação e Comunicação do Programa de Pós-Graduação em Design da Universidade Federal do Maranhão (UFMA), com o intuito de investigar os processos que os alunos do programa seguem para o estudo desse campo. Os resultados foram obtidos através da revisão de literatura de 25 dissertações e identificou-se a predominância de pesquisas que estudam objetos em suporte digital e de abordagem qualitativa. Observou-se também os métodos e técnicas mais utilizados.

Palavras chave Design da Informação, Programa de Pós-Graduação em Design, Mestrado em Design, Universidade Federal do Maranhão. 


\section{Analysis of recurring researches in the dissertations of the Information and Communication line of the Graduate Program in Design at UFMA}

Laís Soares Nehme é Arquiteta e Urbanista graduada pela Universidade Católica de Brasília- UCB (2018). Mestranda em Design, da linha de informação e comunicação, pela UFMA. Graduanda em Engenharia de Software pelo Centro Universitário Dom Bosco- UNDB.

<lais.nehme@discente.ufma.br> ORCID 0000-0001-5620-4879

Melissa Almeida Silva é Arquiteta e Urbanista graduada pela Universidade Estadual do Maranhão (2019). Mestranda em Design pela Universidade Federal do Maranhão na linha de pesquisa de Informação e Comunicação. Atua como Arquiteta e Diretora de Arte com ênfase no projeto de cenários na produção cinematográfica. Atualmente pesquisa o papel do Design na linguagem do cinema.

<melissa.almeida@discente.ufma.br> ORCID 0000-0001-5446-2137
Abstract Information Design (D.I.) has a fundamental role in the transmission of messages of any kind and becomes inherent to citizenship, as its elements and methods have direct consequences on society. The scientific production in this area helps to consolidate it as a scientific field capable of cooperating for the development of society. Considering the importance of the D.I. and its impacts, this article had as its main objective to describe and analyze the dissertations of the research line of Information and Communication of the Post graduate Program in Design at the Federal University of Maranhão (UFMA), in order to investigate the processes that program students follow to study this field. The results were obtained through a literature review of 25 dissertations and identified the predominance of researches that study objects in digital support and with a qualitative approach. The most used methods and techniques were also observed.

Keywords Information Design, Post Graduation Program in Design, Master Program in Design, Federal University of Maranhão.

Análisis de temas recurrentes en las disertaciones de la línea de Información y Comunicación del Programa de Posgrado en Diseño de la UFMA

Resumen El Diseño de la Información (D.I.) tiene un papel fundamental en la transmisión de mensajes de cualquier tipo y se vuelve inherente a la ciudadanía, ya que sus elementos y métodos tienen consecuencias directas en la sociedad. La producción científica en esta área ayuda a consolidarlo como un campo científico capaz de cooperar para el desarrollo de la sociedad. Considerando la importancia del D.I. y sus impactos, este artículo tuvo como objetivo principal describir y analizar las disertaciones de la línea de investigación de Información y Comunicación del Programa de Postgrado en Diseño de la Universidad Federal de Maranhão (UFMA), con el fin de investigar los procesos que los estudiantes del programa siguen para estudiar este campo. Los resultados se obtuvieron a través de una revisión de la literatura de 25 disertaciones e identificaron el predominio de estudios que estudian objetos en soporte digital y con un enfoque cualitativo. También se observaron los métodos y técnicas más utilizados.

Palabras clave Diseño de Información, Programa de Postgrado en Diseño, Maestría en Diseño, Universidad Federal de Maranhão 


\section{Introdução}

Adquirir conhecimento sobre as diferentes abordagens em que um determinado tema é investigado contribui para novos aportes teóricos e metodológicos. Isso se torna ainda mais relevante quando se trata do Design, um campo interdisciplinar com características flutuantes, cuja teoria é constantemente investigada. Dentro dessa temática, o Design de Informação (D.I.) é uma especialidade que alia estudo teórico com prática de projeto, e seus princípios, métodos e impactos na sociedade estão em constante revisão (FARIAS, 2016). Robert E. Horn (1999, p. 15) conceitua esse campo como "a arte e a ciência de planejar informações que podem ser usadas por seres humanos com eficiência e eficácia". Dessa maneira, tendo papel fundamental na transmissão de mensagens de qualquer natureza, o D.I. se torna inerente à cidadania, pois seus elementos e métodos têm consequências diretas na sociedade.

A produção científica na área do D.I. ajuda a consolidá-lo como campo científico apto a cooperar para o desenvolvimento da sociedade. Considerando a importância dessa temática e seus impactos, o presente artigo tem o objetivo de descrever e analisar as dissertações da linha de pesquisa Design: Informação e Comunicação1 do Programa de Pós-Graduação em Design da Universidade Federal do Maranhão (UFMA) por meio de consulta na Biblioteca Digital de Teses e Dissertações da instituição, com o intuito de investigar os processos e caminhos que os alunos do programa seguem para o estudo de D.I. A linha de pesquisa objetiva, em suas diferentes formas, estudar e aplicar métodos e técnicas de pesquisa com foco nos estudos apontados para o design de informação, design de interação, design da comunicação, desenho e cultura visual, design de multimídia e implementação e avaliação de aplicações multimídia interativas.

A ausência do levantamento de dados sobre as dissertações defendidas no programa gera inquietações como: quais as abordagens metodológicas mais recorrentes? Como o D.I. tem sido abordado pelo programa? Quais as contribuições dessas dissertações para o campo? Esses questionamentos realçaram a necessidade de discutir e entender os temas, métodos e técnicas mais comuns. $O$ conhecimento alcançado com esse estudo irá servir como base para rediscutir a linha de pesquisa, apontar as perspectivas e conhecimentos gerados e as lacunas existentes para então planejar futuras atividades e disciplinas. Além disso, tal aprofundamento também irá contribuir para pesquisas sobre o panorama dos estudos em D.I. no Brasil e no Nordeste.

Esse estudo é caracterizado, segundo Romanowski e Ens (2006), como "estado de conhecimento", termo com a mesma natureza de "estado da arte", diferenciando-se por abordar apenas um setor de publicações sobre o assunto estudado. Sobre investigar o estado da arte dos autores reiteram que eles 
(...) podem significar uma contribuição importante na constituição do campo teórico de uma área de conhecimento, pois procuram identificar os aportes significativos da construção da teoria e prática pedagógica, apontar as restrições sobre o campo em que se move a pesquisa, as suas lacunas de disseminação, identificar experiências inovadoras investigadas que apontem alternativas de solução para os problemas da prática e reconhecer as contribuições da pesquisa na constituição de propostas na área focalizada (ROMANOWSKI e ENS, 2006, p. 39).

O programa de pós-graduação em Design da UFMA foi criado em 2012, se consolidando como o terceiro PPG (Mestrado Acadêmico) do Nordeste, com o objetivo de produzir conhecimentos e tecnologias em relação ao desenvolvimento de produtos, por meio de pesquisas descritivas e experimentais. As dissertações apresentadas neste estudo abordam temas, métodos e autores variados, por isso o intuito da pesquisa é elaborar uma síntese para revelar não apenas o histórico das dissertações, mas também a definição de D.I. no programa.

\section{Referencial teórico}

A Escola Superior de Desenho Industrial (ESDI) fundou em 1962 o primeiro curso de graduação em Design no Brasil, e foi considerado modelo na criação e no planejamento de outros cursos em Design no país. No âmbito da pós-graduação stricto sensu (PPGs), Dias (2004) afirma que houve um certo atraso em comparação com outras áreas de conhecimento, sendo criado apenas no início dos anos 90 - notadamente nas regiões Sul e Sudeste -, resultado do amadurecimento dos cursos iniciantes. Os profissionais nesse meio procuraram especializações em outros cursos com áreas de conhecimento que conversam com o design, como ergonomia, comunicação social, engenharia de produção, psicologia, entre outras. Ainda que por falta de opção, essa circunstância contribuiu para o enriquecimento do Design, gerando novos panoramas interdisciplinares (DIAS, 2004, p. 39).

Tal feito veio a colaborar, cada vez mais, com a essência multifacetada do "fazer design". Teses e dissertações nasceram em áreas de concentração e/ou linhas de pesquisa que tivessem algum tipo de afinidade com teoria e práxis do design. Talvez, tal fato possa ser confirmado por Bomfim (2014), o qual relata que a formação do designer apresenta conhecimentos de forma abrangente, mesmo que dissociados e fragmentados, não possuindo um todo homogêneo. Isto pode permitir a este profissional uma gama de possibilidades em conhecimentos de diferentes áreas do saber (DINIZ, 2018, p. 81-82). 
Em 1994, após o primeiro programa de pós-graduação do Brasil stricto sensu em Design ser implantado na PUC-Rio, outras Instituições de Ensino Superior (IES) começaram a se movimentar para a implantação de mestrados e doutorados (TRISKA et al., 2014). Essa disseminação se deu sobretudo no sul do país, e Medeiros (2017) afirma que ainda em 2011, os PPG's eram reduzidos principalmente no Nordeste, que na época apresentava apenas dois programas.

Um ano depois, mais dois novos cursos se consolidaram na região, entre eles o ME na Universidade Federal do Maranhão (UFMA). A implantação do programa foi oportuna e necessária, uma vez que é o terceiro programa de Pós-Graduação (Mestrado Acadêmico) em Design do Norte/Nordeste, o décimo quinto do país e o oitavo a ser ofertado por uma instituição pública Federal no Brasil (DINIZ, 2014). Seu quadro docente é composto por 11 docentes permanentes e 4 colaboradores, tendo formado 76 mestres e se encontra atualmente com 32 mestrandos. Diniz (2018) comenta que a proposta do programa abrangeu a aplicabilidade dos conceitos de design e da sustentabilidade, "além do design e produtos multimídia como um meio de facilitar o uso e o acesso do país como um todo e, principalmente, das demandas da região Norte-Nordeste" (2018, p. 84). O programa vem capacitando e formando mestres em Design de várias regiões do Brasil (Maranhão, São Paulo, Pará, Piauí, Ceará, Goiás) e de outros países (Costa Rica, México, Chile).

As 26 dissertações defendidas no programa abordam temas diversos dentro das linhas de Informação e Comunicação, e identificar essas abordagens de forma sistemática para conhecer o estado da arte, contribui para definirmos os pontos mais estudados do D.I. dos alunos da UFMA. Romanowski e Ens (2006) comentam que esse tipo de estudo aponta a atenção que os pesquisadores estão dando ao tema investigado, além dos aspectos da área do D.I. que mais têm preocupado os pesquisadores. Indicam os temas, subtemas e os tópicos priorizados, e com isso apontam também temas pouco investigados, revelando a necessidade de algumas pesquisas. Os autores seguem afirmando que o levantamento do estado da arte define os aspectos pontuais da proposta específica de um determinado curso ou campo de formação.

Outras questões que esses estudos apontam são os tipos de pesquisas utilizadas, se utilizaram o método pesquisa ação, estudo de caso, etnografia, método heurístico, pesquisa histórica, experimento ou outro método. Indicam as características quanto à abordagem do problema (qualitativa, quantitativa ou quali-quantitativa) e quanto aos objetivos da pesquisa (exploratória, descritiva ou explicativa). Apontam também as técnicas mais utilizadas para a realização dos estudos, se consistem em entrevistas, observação, questionários, análise de documentos, dados coletados por videografia, grupo de discussão, grupo focal, ou outra técnica. (ROMANOWSKI e ENS, 2006, p. 45). 


\section{Métodos}

A natureza da pesquisa caracteriza-se como analítica-descritiva, com abordagem quantitativa e procedimento técnico de revisão de literatura de dissertações da linha de pesquisa de "Design: informação e comunicação" do Programa de Pós-Graduação em Design da UFMA (PPGDg UFMA). Segundo Richardson et al. (1999, p.70), a pesquisa quantitativa se caracteriza pelo uso da quantificação tanto na coleta de dados, quanto no tratamento desses resultados através de técnicas estatísticas. FONTELLES et al. (2009) discorrem sobre as abordagens analítica e descritiva, concluindo que a primeira envolve um estudo mais aprofundado dos dados coletados de um determinado estudo, com o intuito de explicar o contexto de um fenômeno. A de abordagem descritiva tem como intenção observar, registar e descrever as particularidades de um determinado fenômeno ocorrido (LAKATOS e MARCONI, 2001 e 2005 apud. FONTELLES et al. 2009). Sobre a revisão de literatura, Lakatos e Marconi (2005) afirmam que se baseia em uma síntese completa do trabalho e de dados importantes ao tema, dentro de uma sequência lógica (p.248).

Foram realizadas as análises textuais de 25 dissertações da linha, referentes a um período de sete anos, de 2014 a 2020. As dissertações foram acessadas pelo Sistema de Publicação Eletrônica de Teses e Dissertações da UFMA, ou TEDE, a partir de links para as dissertações disponíveis no site do PPGDg UFMA2. Todas as dissertações da linha de pesquisa com links disponíveis para o TEDE foram selecionadas.

Para análise dos dados foram adotados a estatística descritiva e análise semântica de algumas categorias como: tipos de suportes, objetivos da pesquisa, temas, áreas e métodos. Para tal é necessário conhecer tais categorias.

Sobre a categoria suporte, foram classificam em:

- Físico: Artefato que emprega qualidades sensoriais em uma dimensão material.

- Visual: Artefato que emprega qualidades sensoriais em dimensões materiais e virtuais.

- Digital: Artefato que utiliza tecnologia virtual. A interação humana com tal conteúdo é mediada por um computador ou máquina.

Sobre a categoria objetivos, foram classificados em:

- Semântico: Ligado aos significados produzidos pela linguagem por meio da percepção da informação, analisando o aspecto simbólico e a amplitude da mensagem, bem como a eficiência da comunicação.

- Sintático: Ligado à organização espacial da linguagem, relaciona-se ao tamanho, forma, cor e iluminação, com 
o objetivo de facilitar a aquisição das informações pelo usuário, minimizando assim a sua carga cognitiva.

- Pragmático: Ligado à qualidade do uso da linguagem. Contextualiza a linguagem em sua relação com o usuário, analisando possíveis problemas e identificando lacunas.

É possível, em alguns estudos, uma pesquisa empregar mais de um objetivo, como sintático-pragmático, preocupado com a organização e o uso do artefato.

Sobre a categoria de temas e áreas mais abordadas, são definidas em:

- Elementos Visuais: Tratam-se dos elementos que podem ser compreendidos pela visão, abarcando as linguagens pictórica, que compreende imagens, verbal escrita, e esquemática, que integra representações de imagens e textos de maneira integrada.

- Design Gráfico: Trata da manipulação de elementos visuais. Gruszynski (2008) caracteriza a área pela conjunção de signos gráficos e linguísticos, pela produção de peças gráficas que podem ser compostas por fotos, ilustrações, textos e elementos tipográficos, com finalidade de mediar um processo comunicativo.

- Design da Informação: Abarca questões sobre qualidade, objetividade e priorização de informações, além de tratar sobre distribuição e agrupamentos de conteúdos em telas e diferentes suportes (QUARESMA, 2018).

- Design da Interação: Estuda como as interfaces e todos os seus elementos de interação irão funcionar em conjunto de maneira consistente, visando a coerência do todo. É uma área que abrange tanto artefatos físicos quanto digitais. Muitas questões de usabilidade são tratadas nessa área (QUARESMA, 2018).

Sobre a categoria de métodos mais abordados, são definidos em:

- Estudo de Caso: Caracteriza-se por contribuir para a compreensão que temos de fenômenos políticos, sociais e organizacionais, colocando questões do tipo "como e por que", onde o pesquisador tem pouco controle sobre os fatos e seu foco se encontra em acontecimentos contemporâneos enquadrado em alguma circunstância da vida real YIN (2010). Esse método é usado 
frequentemente na produção de conhecimento das Ciências Sociais.

- Teste de Usabilidade: Utiliza representantes de um público-alvo como participantes de testes, a fim de avaliar até que ponto um produto atende a critérios de usabilidade específicos (RUBIN e CHISNELL, 2008).

\section{Resultados}

Identificou-se que 22 das 25 dissertações analisadas adotaram suportes de objetos de estudo digitais, ao passo que 2 dissertações adotaram suportes visuais e 1 adotou suporte físico, o que delineia o perfil de uso de suportes digitais na linha.

Através da análise das relações entre parâmetros de objetivos e abordagens, constata-se a predominância de abordagens qualitativas em dissertações com objetivos sintáticos, o que pode indicar um perfil de produção de conhecimento não generalizável para pesquisas com tais objetivos. Em geral, os pesquisadores da linha tendem a optar por abordagens qualitativas. Contudo, percebe-se que as pesquisas que adotaram abordagens quantitativas e mistas são predominantemente pragmáticas. A tabela 1 explora tais relações, evidenciando ainda a predominância de pesquisas com objetivos sintáticos e pragmáticos.

\begin{tabular}{lcccc} 
Objetivos & Abordagens & & \\
\hline & Mista & Qualitativa & Quantitativa & Total \\
\hline Semântico & 1 & 1 & 1 \\
\hline Sintático & 2 & 10 & 2 & 11 \\
\hline Pragmático & 8 & & 12 \\
\hline Sintático-Pragmático & 2 & 1 & 3 & 1 \\
\hline Total Abordagens & 20 & 25 \\
\hline
\end{tabular}

Tabela 1. Relações entre abordagens

e objetivos de pesquisa.

Fonte: Os autores, 2021
A tabela 2 apresenta os termos mais citados dos títulos das dissertações organizados em categorias. Analisando os termos das categorias "suporte" e "temas/áreas" verifica-se que não há um termo que mais se destaca, contudo, o campo semântico está muito próximo, envolvendo a interação de interfaces digitais. Na categoria "outros" percebe que há uma valorização da experiência do usuário. 


\begin{tabular}{|c|c|c|c|c|c|c|c|}
\hline Suporte & & Temas/Área & & Métodos & & Outros & \\
\hline Digital & 5 & Interação & 5 & Análise & 8 & Usuário & 6 \\
\hline Interface & 4 & Aplicativo & 5 & Estudo de caso & 5 & Processo & 4 \\
\hline Visual & 3 & Usabilidade & 4 & Avaliação & 4 & Experiência & 4 \\
\hline
\end{tabular}

Tabela 2. Temas abordados

na linha de pesquisa.

Fonte: Os autores, 2021

Fig 1. Nuvem de palavras

Fonte: Os autores, 2021

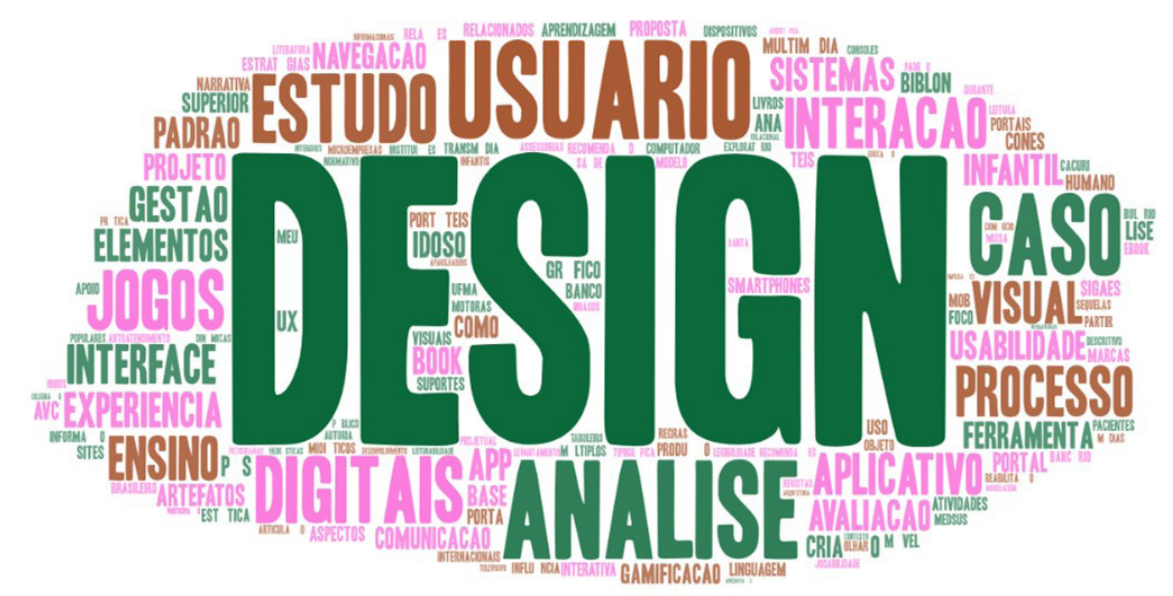

Com a nuvem de palavras emerge mais termos que a tabela 2 não revela como jogos, ensino, entre outros. De qualquer forma, o título não apresenta toda a complexidade das pesquisas, com seus temas e métodos, contudo apontam para algumas questões que podem ser constatadas com outros dados.

A tabela 3 apresenta as principais áreas de pesquisa trabalhadas dentro da linha através das palavras-chaves da pesquisa. Alguns pesquisadores optaram por trabalhar com mais de uma área em suas pesquisas, o que justifica o somatório de frequências ser superior a 25 , número total de dissertações analisadas.

\begin{tabular}{ll}
\hline Áreas & $\mathbf{f}$ \\
\hline Design da Interação & 11 \\
\hline Design Gráfico & 7 \\
\hline Design da Informação & 5 \\
\hline Elementos Visuais & 4 \\
\hline Outros & 6 \\
\hline
\end{tabular}

Tabela 3. Áreas trabalhadas dentro da linha de pesquisa Fonte: Os autores, 2021 
Análise de temas recorrentes nas dissertações da linha de Informação e

Comunicação do programa de Pós-Graduação em Design da UFMA

A principal palavra-chave mencionada é "design de interação" que se coaduna com as categorias "suporte" e "temas/áreas" dos títulos, podendo inferir que a área de design interação teria um peso maior do que a área de design de informação.

Os principais autores citados podem indicar as principais referências teóricas. Os principais autores citados foram:

\begin{tabular}{|c|c|c|c|}
\hline & Áreas & Autores & f \\
\hline & \multirow[t]{3}{*}{ Dados Nacionais } & Brasil & 14 \\
\hline & & Sebrae & 07 \\
\hline & & ABNT & 04 \\
\hline & \multirow[t]{11}{*}{ Interação/Interface } & Preece, Rogers e Sharp & 14 \\
\hline & & Cybis & 13 \\
\hline & & Padovani & 11 \\
\hline & & Nilsen & 11 \\
\hline & & Norman & 08 \\
\hline & & Teixeira & 06 \\
\hline & & Paulino & 05 \\
\hline & & Stumpf & 05 \\
\hline & & Barbosa, Silva & 04 \\
\hline & & Santa Rosa & 04 \\
\hline & & Dick & 04 \\
\hline & \multirow[t]{4}{*}{ Metodologia } & Gil & 13 \\
\hline & & Prodanov & 06 \\
\hline & & Yin & 05 \\
\hline & & Lakatos e Marconi & 05 \\
\hline & \multirow[t]{4}{*}{ Gráfico/Tipografia } & Lupton & 11 \\
\hline & & Samara & 07 \\
\hline & & Bringhurst & 07 \\
\hline & & Ambrose & 05 \\
\hline & \multirow[t]{3}{*}{ Informação } & Agner & 08 \\
\hline & & Spinillo & 07 \\
\hline & & Filatro & 05 \\
\hline & \multirow[t]{5}{*}{ História e Teoria } & Bonsiepe & 13 \\
\hline & & Obregon & 10 \\
\hline & & Cardoso & 06 \\
\hline & & Santaella & 05 \\
\hline & & Crossan & 04 \\
\hline \multirow{2}{*}{$\begin{array}{l}\text { Tabela 4. Autores e referências } \\
\text { mais utilizadas } \\
\text { Fonte: Os autores, } 2021\end{array}$} & & Lévy & 04 \\
\hline & & Manzini & 04 \\
\hline
\end{tabular}


Foram mais de 1.500 referências, uma média de 60,1 referências por pesquisa. Algumas referências foram:

- Dados Nacionais: são os dados ou normais do país como questões sociais, econômicas e perfil da população;

- Interação/Interfaces: é a área com mais autores citados que são utilizados para definir conceitos e métodos, como Preece, Rogers e Sharp, que são autores do livro "Design de Interação: além da Interação HumanoComputador", que apresenta uma perspectiva prática, interdisciplinar e orientada a processo, revelando como eles podem ser aplicados ao design de interação;

- Metodologia: são autores que discutem os métodos e técnicas de pesquisa. O principal autor citado foi o Gil, autor do livro "Como Elaborar projetos de pesquisa", que oferece condições para organização de conhecimentos variados tanto para estudantes, quanto para profissionais de pesquisa;

- Design Gráfico/Tipografia: é a área que estudo os elementos visuais gráficos e suas disposições no suporte visual. Como autora principal a Samara, designer gráfico e autor de livros como "Grid" e "Evolução de design";

- Informação: é a área preocupada na estruturação visual da informação. Como autor principal a Agner, autor de diversas publicações da área.

- História e Teoria: campo com referenciais teóricos e históricos sobre o Design e a Comunicação. O principal autor é Bonsiepe, com diversas publicações, a mais citada foi "Design, Cultura e Sociedade".

A tabela 5 explicita os métodos de pesquisa mais utilizados nas dissertações da linha. Muitos pesquisadores combinaram diferentes métodos em suas pesquisas, o que justifica o somatório de frequências de métodos ser superior a 25 , número total de dissertações analisadas. Identificou-se que 19 pesquisadores optaram por coletar dados com participantes, enquanto 6 pesquisadores optaram por não fazê-lo. Em pesquisas que adotaram o método "Estudo de Caso", predominante na linha, 11 entre 14 dissertações coletaram dados com participantes.

É pertinente ressaltar que os métodos indicados na tabela foram informados pelos pesquisadores. Nesse sentido, alguns autores descrevem as técnicas utilizadas em suas pesquisas, mas não os métodos. Três autores descreveram procedimentos similares aos do método "Heurístico". Um autor utiliza o termo «Ensaio de Interação», cuja descrição se assemelha ao 
"Teste de Usabilidade". Métodos de inspeção, como "Avaliação Heurística", "Inspeção Semiótica" e "Percurso Cognitivo", que Barbosa e Silva (2010) recomendam utilizar para antever consequências das decisões de projetos de design ainda não implementados sobre a usabilidade, eventualmente foram utilizados para avaliar sistemas que já são utilizados por usuários.

Como já mencionado, o método mais utilizado pelos pesquisadores nas dissertações foi o "Estudo de Caso". Pode-se relacionar a frequência de uso desse método com o número de dissertações com a abordagem qualitativa (tabela 1), onde as pesquisas desse tipo são 20 , representando grande parte dos trabalhos. Tal análise é reforçada pela argumentação de Pádua (2004), que afirma que o "Estudo de Caso" é de abordagem qualitativa, seja como atividade complementar de uma coleta de dados, ou como próprio trabalho monográfico (PÁDUA, 2004 apud. ORSOLINE e OLIVEIRA, 2013).

O "Teste de Usabilidade" foi utilizado em 5 dissertações, o que pode justificar o número de pesquisas com objetivos pragmáticos (tabela 1), tendo em vista que esse tipo de abordagem trabalha com o usuário.

Os outros métodos utilizados se dividiram em "Avaliação Heurística”, "Teste de produção", "Delphi”, "Etnografia", "Inspeção Semiótica", "Percurso Cognitivo" e "Pesquisa de Campo". Muitos pesquisadores combinaram diferentes técnicas em suas pesquisas, o que justifica o somatório de frequências de técnicas ser superior a 25, número total de dissertações analisadas. As técnicas utilizadas na linha são contabilizadas na tabela 5.

Tabela 5. Métodos mais utilizados

Métodos

Fonte: Os autores, 2021

\begin{tabular}{lc}
\hline Estudo de Caso & 14 \\
\hline Teste de Usabilidade & 5 \\
\hline Outros & 9 \\
\hline Total & 28 \\
\hline
\end{tabular}

A tabela 6 explicita as técnicas de pesquisa mais utilizadas nas dissertações da linha. Muitos pesquisadores combinaram diferentes técnicas em suas pesquisas, o que justifica o somatório de frequências de técnicas ser superior a 25, número total de dissertações analisadas.

o levantamento das técnicas mais frequentes reafirma o perfil da linha em relação a coleta de dados com participação de usuários: apenas duas das técnicas utilizadas, inspeção e observação sistemática, não requerem a participação dos usuários. Ainda assim, 14 dentre as 18 pesquisas que utilizaram a "Observação Sistemática" também optaram por coletar dados com participantes. 


\begin{tabular}{lc} 
Técnicas & $\mathbf{f}$ \\
\hline Observação Sistemática & 18 \\
\hline Questionários & 9 \\
\hline Entrevistas & 6 \\
\hline Grupo Focal & 3 \\
\hline Inspeção & 2 \\
\hline $\begin{array}{l}\text { Teste de estimativa de } \\
\text { compreensibilidade }\end{array}$ & 50 \\
\hline Total & 2
\end{tabular}

\section{Conclusão}

Realizou-se uma revisão de literatura de 25 dissertações, defendidas entre 2014 e 2020, da linha de pesquisa de "Design: informação e comunicação" do PPGDg UFMA, com o objetivo de identificar o estado do conhecimento da linha de pesquisa. Para tanto, foram avaliados os parâmetros: classificação de objetivos das pesquisas, abordagens utilizadas, temas frequentes, áreas frequentemente trabalhadas, métodos e técnicas de pesquisa, além de autores mais citados por área do conhecimento.

Identificou-se a predominância de pesquisas que estudam objetos em suporte digital, além da predominância da abordagem qualitativa nas pesquisas. Os métodos "Estudo de Caso" e "Teste de Usabilidade" são os mais utilizados na linha, junto às técnicas de "Observação Sistemática" e "Questionários".

O programa aborda a Comunicação e Informação majoritariamente a partir das áreas de pesquisa "Design da Interação", "Design Gráfico" e "Design da Informação", os termos, métodos e autores utilizados refletem o foco da linha em temas de interface e interação. Os objetivos de pesquisa mais explorados nas dissertações são sintáticos e pragmáticos. Essas características dos trabalhos da linha podem representar um reflexo dos conteúdos ministrados nas disciplinas "Tópicos especiais em Design da Informação" e "Design e Interação Humano-Computador" do mestrado.

Em geral, as dissertações da linha têm contribuído com análises da configuração visual de diferentes artefatos de suporte digital, no sentido de facilitar a apreensão da informação pelo usuário. Além disso, as dissertações têm explorado ainda a experiência de uso de artefatos digitais, frequentemente contando com a participação de usuários. Portanto, seria interessante refletir sobre a possibilidade de modificar o nome da linha para expressar o foco da mesma em interface e interação. 
Contudo, por mais que o "Design da Interação" seja a área mais pesquisada na linha, percebe-se que métodos inspecionais, como "Avaliação Heurística" e "Percurso Cognitivo" foram pouco utilizados nas pesquisas da linha, o que pode representar uma lacuna a ser preenchida pela disciplina "Design e Interação Humano-Computador".

Por fim, se esclarecem as limitações do presente estudo, uma vez que as dissertações da linha de pesquisa "Design: informação e comunicação" do PPGDg UFMA não foram todas contempladas nesta análise, devido a restrições de acesso a algumas dissertações no site do programa, foram analisadas 25 das 29 dissertações listadas no site.

\footnotetext{
1. 0 programa tem como área de concentração Design de produtos e três linhas de pesquisa. Sendo as outras Design: materiais, processos e tecnologia e Design: ergonomia e usabilidade de produtos e sistemas.

2 PPGDg UFMA. Dissertações defendidas. Disponível em: http:// www.ppgdg.ufma.br/dissertacoes-defendidas/

Acesso em: 18 jul 2021.
}

\section{Referências}

BARBOSA, Simone; SILVA, Bruno. Interação humano-computador [recurso eletrônico]. Rio de Janeiro: Elsevier, 2010.

DIAS, Maria Regina Álvares Correia. 0 ensino do Design: A interdisciplinaridade na disciplina de projeto em Design. Orientador: Leila Amaral Gontijo. 2004. 163 p. Dissertação (Mestrado em Engenharia de Produção) - Universidade Federal de Santa Catarina, Florianópolis, 2004.

DINIZ, Raimundo Lopes. Pós-Graduação em Design no Brasil: especificidades das regiões Norte e Nordeste. Diálogo com a Economia Criativa, Rio de Janeiro, v. 3, n. 7, p. 80-93, 14 abr. 2018

DINIZ, R. L. A Pós-Graduação em Design no Brasil: proposta de mestrado oferecida pela Universidade Federal do Maranhão (UFMA). Estudos em Design | Revista (online). Rio de Janeiro: v. 22 |n. 3 [2014], p. 57 - 69| ISSN 1983-196X.

FARIAS, P. L. (2016). Histórias e Teorias do design da informação. Revista Brasileira de Design da Informação, v. 13 n. 2. São Paulo.

FONTELLES, Mauro José; SIMÕES, Marilda Garcia; FARIAS, Samantha Hasegawa; FONTELLES, Renata Garcia Simões. Metodologia para a pesquisa científica: diretrizes para a elaboração de um protocolo de pesquisa. Núcleo de Bioestatística Aplicado à Pesquisa da Universidade da Amazônia - UNAMA, Belém, p. 1-8, 2009. 
GRUSZYNSKI, Ana Cláudia. Design Gráfico: entre o invisível e o ilegível. São Paulo: Edições Rosari, 2008.'HORN, R. E. (1999). Information Design: Emergence of a New Profession. Em R. Jacobson (Ed.). Information Design. Cambridge, Massachusetts. London.England. Massachusetts Institute of Technology: The MIT Press.

MARCONI, M. A. e LAKATOS, E. M. Fundamentos de metodologia científica. $6^{a}$ ed. São Paulo, SP: Atlas, 2005.

MEDEIROS, W. G. Graduação e pós-graduação em design na Paraíba: breve relato sobre os fatores de criação dos cursos de bacharelado e mestrado em design na UFCG. In: Revista de Ensino em Artes, Moda e Design. Universidade do Estado de Santa Catarina. Centro de Artes. Programas de Pós-Graduação em Artes, design e consumo da PPGAV/ UDESC, ICA/UFC, PPGD/ UFPE e PGCDS/ UFRPE. n. 1, Florianópolis: UDESC/CEART, 2017. Pp. $65-82$

ORSOLINI, A. V. P. ; OLIVEIRA, Sheila Fernandes Pimenta e . Estudo de Caso como método de investigação qualitativa: uma abordagem bibliográfica. In: Bárbara Fadel; Marinês Santana Justo Smith. (Org.). CULTURA E DESENVOLVIMENTO - Série: Desenvolvimento Regional em Perspectiva. 1 ed.Franca: Centro Universitário de Franca - Uni-FACEF, 2013, v. 1, p. 01-18

PALMA, Lorena Cristina Romero; GUARNIERI, Patricia Vecchio; PANSERA, Fernanda Cristina; GATTI, Sandra Regina Teodoro. Um levantamento de teses e dissertações de um programa de pós-graduação sobre a aproximação da História da Ciência e o Ensino de Ciências. ACTIO: Docência em Ciências, Curitiba, v. 2, n. 1, p. 401-419, 1 jul. 2017.

QUARESMA, Manuela; "UX Designer: quem é este profissional e qual é a sua formação e competências?”. In: Design para acessibilidade e inclusão. São Paulo: Blucher, 2018. p. 88 -101 RICHARDSON, Roberto Jarry et al. Pesquisa Social: métodos e técnicas. 3 ed. São Paulo: Atlas, 1999.

ROMANOWSKI, J. P.; ENS, R. T. As pesquisas denominadas do tipo "estado da arte" em educação. Diálogo Educacional, v. 6, n. 19, p. 37-50, set./dez., 2006.

SANTOS, Aguinaldo dos. Seleção do método de pesquisa: guia para pós-graduandos em design e áreas afins. Curitiba, PR: Insight, 2018. 230p.

TRISKA, R., VELA, J. C., DOLZAN, J. E. A pós-graduação stricto sensu do Design no Brasil: uma leitura. Estudos em Design | Revista (online). Rio de Janeiro: v. 22 | n. 3 [2014], p. 70 - 80 | ISSN 1983-196X

YIN, R. K. Estudo de Caso: planejamento e métodos. 4. ed. Porto Alegre: Bookman, 2010. 